\title{
Pulmonary sclerosing pneumocytoma with multiple satellite nodules mimicking tuberculosis
}

\author{
Min A Lee ${ }^{1}$, Tae Jung Kim ${ }^{1}$, Joungho Han ${ }^{2}$, Kyung Soo Lee ${ }^{1}$ \\ ${ }^{1}$ Department of Radiology and Center for Imaging Science, Samsung Medical Center, Sungkyunkwan University School of \\ Medicine, Seoul, Korea \\ ${ }^{2}$ Department of Pathology, Samsung Medical Center, Sungkyunkwan University School of Medicine, Seoul, Korea
}

Received: May 7, 2018

Revised: May 21, 2018

Accepted: May 29, 2018

Corresponding author:

Tae Jung Kim

Department of Radiology and Center for Imaging Science, Samsung Medical Center, Sungkyunkwan University School of Medicine, 81 Irwonro, Gangnam-gu, Seoul 06351, Korea

Tel: +82-2-3410-0715

E-mail: taejung.kim1@gmail.com

\section{ABSTRACT}

Pulmonary sclerosing pneumocytoma is a rare, benign lung tumor commonly seen in women in the fifth decade of life. It usually manifests as a solitary, well-circumscribed juxtapleural nodule or mass with intense, homogeneous enhancement on computed tomography scans. Herein, we report a case of sclerosing pneumocytoma presenting as main nodule with satellite nodules, mimicking tuberculosis.

Keywords: Satellite nodules; Sclerosing pneumocytoma; Tomography; Tuberculosis

\section{INTRODUCTION}

Sclerosing pneumocytoma (previously called sclerosing hemangioma) is a rare, benign tumor of the lung with uncertain histogenesis. It is usually seen in middle-aged women [1]. Sclerosing pneumocytoma is considered as a type of benign epithelial tumor arising from type II pneumocytes. Sclerosing pneumocytoma usually manifests as a solitary, well-circumscribed juxtapleural nodule or mass with intense, homogeneous enhancement on computed tomography (CT) scans due to its hemangiomatous component [2]. The occurrence of multiple tumors or as a tumor surrounded by multiple satellite nodules within the same lobe is rare [2,3]. Herein, we report a case of sclerosing pneumocytoma presenting as main nodule with surrounding satellite nodules mimicking tuberculosis.

\section{CASE REPORT}

This is an Open Access article distributed under the terms of the Creative Commons Attribution Non-Commercial License (http:// creativecommons.org/licenses/ by-nc/4.0/).
A 23-year-old woman visited a local hospital with history of blood-tinged sputum for 5 days. She had no previous history of tuberculosis and was a non-smoker. Her chest radiograph revealed a nodular opacity with satellite nodules in the mid zone of the right lung (Fig. 1A). Following this, she was referred to our hospital for further evaluation. CT performed with intravenous contrast revealed a $25-\mathrm{mm}$ lobulated nodule with heterogeneous contrast enhancement, 


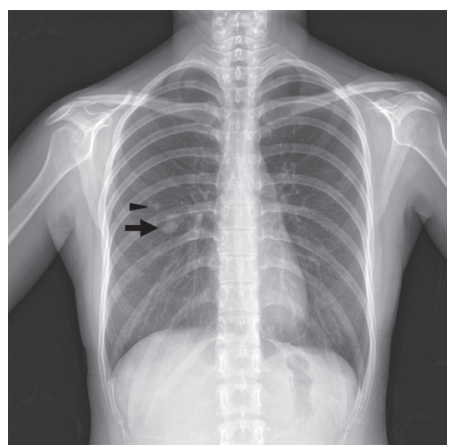

A
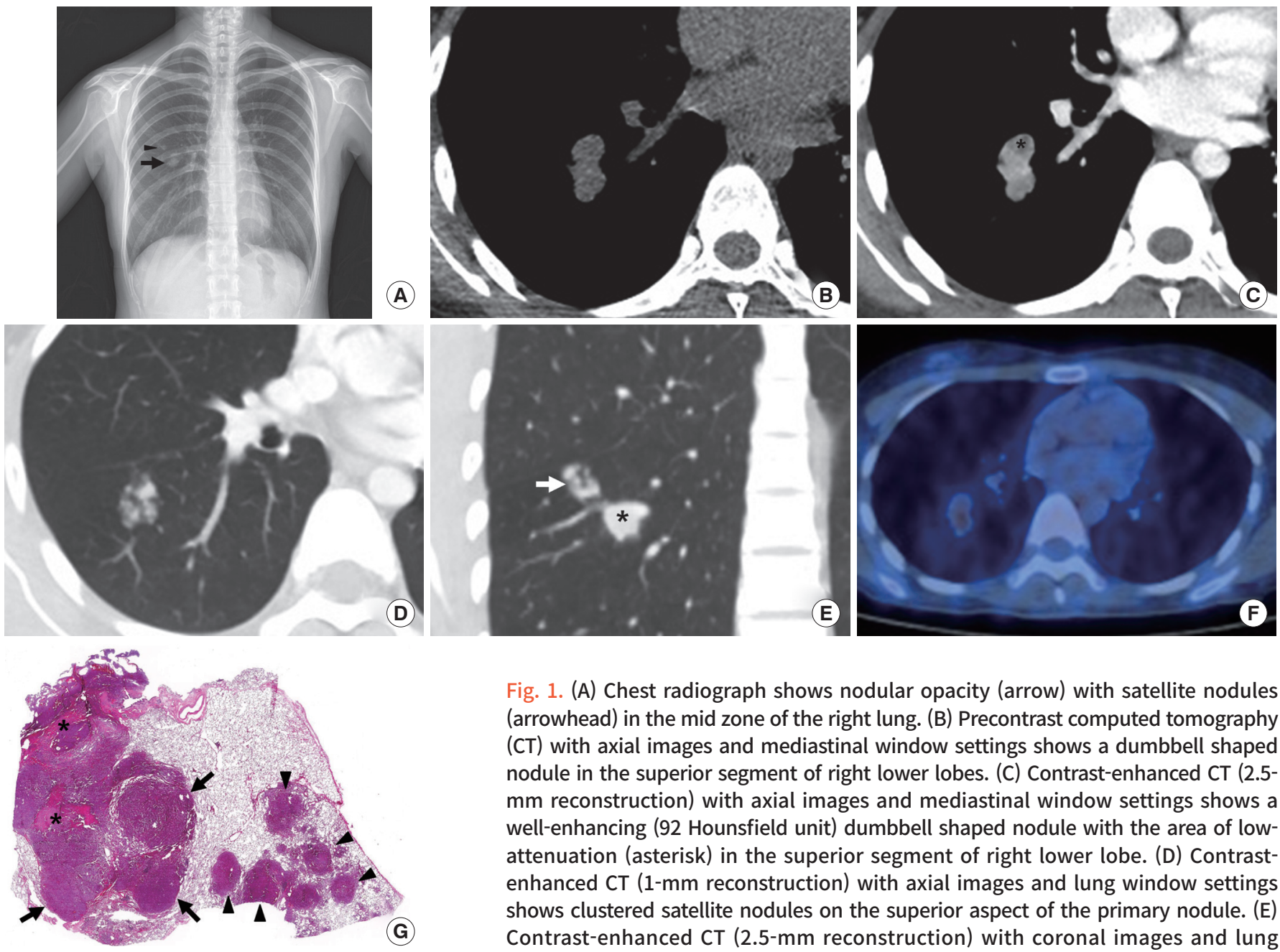

Fig. 1. (A) Chest radiograph shows nodular opacity (arrow) with satellite nodules (arrowhead) in the mid zone of the right lung. (B) Precontrast computed tomography (CT) with axial images and mediastinal window settings shows a dumbbell shaped nodule in the superior segment of right lower lobes. (C) Contrast-enhanced CT (2.5$\mathrm{mm}$ reconstruction) with axial images and mediastinal window settings shows a well-enhancing (92 Hounsfield unit) dumbbell shaped nodule with the area of lowattenuation (asterisk) in the superior segment of right lower lobe. (D) Contrastenhanced CT (1-mm reconstruction) with axial images and lung window settings shows clustered satellite nodules on the superior aspect of the primary nodule. (E) Contrast-enhanced CT (2.5- $\mathrm{mm}$ reconstruction) with coronal images and lung window setting shows a main nodule (asterisk) with satellite clustered nodules

(white arrow) in the superior segment of right lower lobe. (F) ${ }^{18} \mathrm{~F}$-fluorodeoxygloucose positron emission tomography CT reveals that the maximum standardized uptake value of the nodule in the right lower lobe is 2.4 and no other hypermetabolic lesion was detected. (G) Light microscopic image shows a primary nodule consisting of predominantly hypercellular solid component (arrows) and hemorrhagic hemangiomatous component (asterisks) with several satellite nodules (arrowheads) consisting of predominantly solid component in the normal lung parenchyma (H\&E stain, $\times 40)$.

located in the superior segment of the right lower lobe with clustered satellite nodules on the superior aspect of the primary nodule (Fig. 1B-E). The patient's laboratory findings were within normal limits. Based on the $\mathrm{CT}$ findings, active pulmonary tuberculosis was suspected. Tests for acid-fast bacilli (AFB) via sputum culture and Mycobacterium tuberculosis polymerase chain reaction returned negative results. However, considering her $\mathrm{CT}$ findings and residence in an endemic area for tuberculosis, we initiated a standard "short" anti-mycobacterial regimen including isoniazid, rifampin, pyrazinamide, and ethambutol. On follow-up 2 months later, there was no recurrence of blood-tinged sputum production; however, chest CT showed no interval change of a main nodule with clustered satellite nodules. Further follow-up at 6 months after treatment did not reveal any change in the CT findings. Hence, the pulmonologist performed a biopsy of the 25-mm main nodule using radial probe endobronchial ultrasound. Histopathological examination of the bronchoscopic biopsy specimen did not reveal a granuloma or fungal lesion. AFB culture of the tissue specimens was negative. A whole body ${ }^{18} \mathrm{~F}$-fluorodeoxyglucose positron emission tomography-CT exam was performed. The maximum standardized uptake value of the nodule in the right lower lobe was 2.4 (Fig. 1F) and no other hypermetabolic lesion was detected. Finally, we decided on video-assisted thoracoscopic surgery with superior segmentectomy of the right lower lobe under joint consultation of the pulmonologist, the radiologist, and the thoracic surgeon. The histopathological features 
PRECISION AND FUTURE MIEDICINE

Sclerosing pneumocytoma with multiple nodules

demonstrated sclerosing pneumocytoma with several satellite nodules (Fig. 1G).

\section{DISCUSSION}

Sclerosing pneumocytoma is a rare tumor of the lung that is thought to arise from type II pneumocytes of the respiratory epithelium. This tumor usually occurs in middle-aged adults, with a male-to-female ratio of 1:5 [4]. Most patients are asymptomatic; however, some show clinical manifestations, including cough, hemoptysis, chest pain, and stuffiness [5]. These tumors are composed of two cell-types with cuboidal epithelial lining cells and round cells with either eosinophilic or clear cytoplasm forming sheets. They are of four major histologic patterns: hemangiomatous, papillary, sclerotic, and solid, and are associated with various findings on CT scans according to composition [2,6]. On CT-pathology correlation, areas of hyper-, iso-, and hypoattenuation correspond to angiomatous, solid, and sclerotic histologic patterns, respectively [7]. Based on reports of several CT findings till date, the morphologic characteristics of sclerosing pneumocytoma include a solitary juxtapleural mass, circular or ovoid in shape, with smooth margins and a diameter of $2.2 \pm 0.3 \mathrm{~cm}[2,5,8]$. The mean tumor attenuation value on CT is $30 \pm 3$ Hounsfield unit (HU) before intravenous administration of contrast media [7]. After intravenous contrast, the mass reveals intense, homogeneous enhancement, with maximum attenuation values ranging from 90 to $110 \mathrm{HU}[2,7]$.

In our case, the initial diagnosis considered was an inflammatory lesion, such as tuberculosis or aspergillosis, given the presentation of a heterogeneous primary nodular lesion combined with clustered satellite nodules in a young woman. The probability of a tumor was considered low based on the clinical and radiological findings. The presence of satellite nodules, dumbbell shape, and heterogeneous enhancement was not characteristic of sclerosing pneumocytoma; however, the intense enhancement on contrast CT of $92 \mathrm{HU}$ was suggestive. Sclerosing hemangioma has been reported to manifest as multiple tumors in up to $4 \%$ of cases $[9,10]$. However, there has only been one case reported so far, manifesting as clustered nodules with a foci of ground-glass opacity, localized in a lung lobe [3]. This case was also initially diagnosed as active pulmonary tuberculosis.

In conclusion, sclerosing pneumocytoma generally manifests as a solitary, well-circumscribed nodule or mass. Although very rare, it can present main nodule with surround- ing clustered satellite nodules on $\mathrm{CT}$ imaging, mimicking active pulmonary tuberculosis.

\section{CONFLICTS OF INTEREST}

No potential conflict of interest relevant to this article was reported.

\section{REFERENCES}

1. Baysak A, Oz AT, Mogulkoc N, Bishop PW, Ceylan KC. A rare tumor of the lung: pulmonary sclerosing hemangioma (pneumocytoma). Respir Med 2013;107:448-50.

2. Chung MJ, Lee KS, Han J, Sung YM, Chong S, Kwon OJ. Pulmonary sclerosing hemangioma presenting as solitary pulmonary nodule: dynamic CT findings and histopathologic comparisons. AJR Am J Roentgenol 2006;187: 430-7.

3. Shin SY, Kim MY, Lee HJ, Oh SY, Jang SJ. Clustered pulmonary sclerosing pneumocytoma in a young man: a case report. Clin Imaging 2014;38:532-5.

4. Devouassoux-Shisheboran M, Hayashi T, Linnoila RI, Koss MN, Travis WD. A clinicopathologic study of 100 cases of pulmonary sclerosing hemangioma with immunohistochemical studies: TTF-1 is expressed in both round and surface cells, suggesting an origin from primitive respiratory epithelium. Am J Surg Pathol 2000;24:906-16.

5. Wang QB, Chen YQ, Shen JJ, Zhang C, Song B, Zhu XJ, et al. Sixteen cases of pulmonary sclerosing haemangioma: $\mathrm{CT}$ findings are not definitive for preoperative diagnosis. Clin Radiol 2011;66:708-14.

6. Kim GY, Kim J, Choi YS, Kim HJ, Ahn G, Han J. Sixteen cases of sclerosing hemangioma of the lung including unusual presentations. J Korean Med Sci 2004;19:352-8.

7. Im JG, Kim WH, Han MC, Han YM, Chung JW, Ahn JM, et al. Sclerosing hemangiomas of the lung and interlobar fissures: CT findings. J Comput Assist Tomogr 1994;18:34-8.

8. Shin SY, Kim MY, Oh SY, Lee HJ, Hong SA, Jang SJ, et al. Pulmonary sclerosing pneumocytoma of the lung: $\mathrm{CT}$ characteristics in a large series of a tertiary referral center. Medicine (Baltimore) 2015;94:e498.

9. Shimosato Y. Lung tumors of uncertain histogenesis. Semin Diagn Pathol 1995;12:185-92.

10. Hishida T, Yoshida J, Nishimura M, Ishii G, Nishiwaki Y, Nagai K. Multiple sclerosing hemangiomas with a 10-year history. Jpn J Clin Oncol 2005;35:37-9. 